\title{
Clinical and Microbiological Evaluation of an Extended-Wear Hearing Instrument
}

\author{
Jae Hoon Sim ${ }^{a} \quad Y^{c}$ ouzhou Xie ${ }^{a} \quad$ Erdal Karamuk ${ }^{c} \quad$ Alexander von Kameke ${ }^{c}$ \\ Jens Ten Holder ${ }^{c}$ Reinhard Zbinden ${ }^{b} \quad$ Christof Rööslia Alexander M. Huber ${ }^{a}$ \\ a University Hospital Zurich, and ${ }^{b}$ Institute of Medical Microbiology, University of Zurich, Zurich, \\ and ${ }^{\mathrm{C}}$ Phonak AG, Stäfa, Switzerland
}

Key Words

Extended wear · Hearing aid · Ear canal skin · Microbiology · Pathology

\begin{abstract}
This study aimed to examine the integrity of the ear canal skin and its microbiological changes caused by an extended-wear hearing device. An extended-wear hearing device, Lyric2 (Phonak AG, Switzerland), which is the second generation of the Lyric device, was fitted to 52 ears of 27 subjects. A refit of the device was done for a selection of ears $(n=15)$ for which the initial fitting was interrupted (28 ears). A matching control group consisted of 10 ears of 10 subjects with no history of using any form of hearing aid for 3 months. The ear canal skin of ears fitted with the hearing device was inspected using an otoendoscope, and signs of problematic pathological conditions were graded. Microbiological examinations were performed at three different stages following fitting. Wearing the device was interrupted in 28 (53.8\%) out of the 52 ears for the initial fitting, and 17 of the 28 interrupted cases $(60.7 \%)$ were due to issues of the ear canal skin. In 3 of 15 ears (25\%) following a refitting, wearing the device was interrupted yet again, and 1 of these 3 interruptions was caused by ear canal skin pathologies. Growth of bacteria was not enhanced or prompted by wearing the Lyric2, and Gram-negative bacteria could not be cultured while the device was in place. Based on aspects of ear canal skin pathology, continuous and nonproblematic uses of the Lyric2 device are presumed to be obtained if the fitting is done carefully. Wearing of the Lyric2 device can be considered safe from a microbiological perspective and does not promote growth of Gram-negative bacteria.
\end{abstract}




\section{Introduction}

While various types of hearing aids have provided benefits to individuals with hearing loss, most current hearing aids still have drawbacks such as dissatisfaction with the quality of sound, the occlusion effect (autophony), comfort, and visibility [Branda, 2012]. In addition, people often object to the daily routines associated with hearing aid use such as daily insertion and removal, replacement of batteries, telephone and earphone usage, wind noise, and problems associated with cerumen [Kochkin, 2007].

In an attempt to resolve such traditional drawbacks of hearing aids, extended-wear hearing devices have been designed. These are positioned deep into the external auditory canal (e.g. Lyric, Phonak AG, Switzerland; fig. 1). Insertion of such a device is usually performed by a trained hearing health care professional, and the device remains in situ for up to 120 days without further maintenance. Positioned millimeters laterally from the tympanic membrane, the device sits mostly in the bony part of the external auditory canal. The benefits of the extended-wear hearing device include its total invisibility, its ability to provide continuous full-time hearing support without daily handling and maintenance such as regular cleaning or battery exchange, minimal occlusion due to its deep placementin the ear canal [Gudmundsen, 1994; Branda, 2012], and the ability to hear during sleep.

Such extended-wear hearing devices need to fulfill various requirements with regard to biocompatibility and pressure applied to the skin as well as environmental properties such as breathability and moisture transport in order to maintain a healthy physiological condition of the ear canal. Fitting and wearing the extended-wear hearing device may cause changes of the physiological status of the ear canal skin, possibly leading to discomfort, at which point the subject, along with professional guidance, must decide if continuation of device use is warranted. Further, if significant problems are observed on the ear canal skin, the device should be removed even if the subject is not feeling discomfort.

One specific difference between a conventional and an extended-wear hearing instrument is that with the latter, the external ear canal is continuously occluded. Occlusion by a device may increase the relative humidity of the inner part of the auditory canal which in turn may

Fig. 1. Schematic visualization of the deep-fitted Lyric2 device (Phonak AG, Switzerland) in the ear canal.

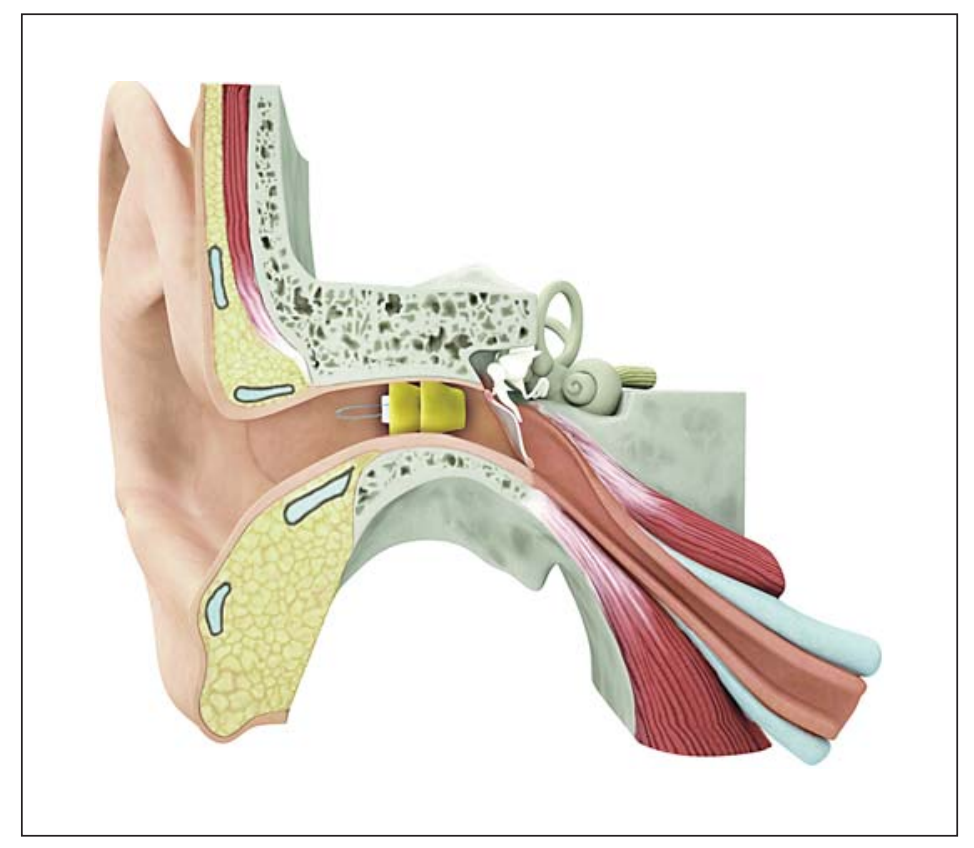


make the area more prone to infection. It has been shown that occlusion of the ear canal by conventional hearing aids does increase the number of microorganisms, and an increase in the number of pathogenic bacteria accompanied by skin maceration could promote an infection [Brook and Coolbaugh, 1984; Karaca et al., 2013]. Although more open-fit hearing aids allow a certain amount of humidity transport and compensation of static pressure changes, it is still questionable whether occlusion by any portion of the hearing device affects the microbiology in the ear canal.

This study aimed to investigate issues related to ear canal health in subjects fitted with an extended-wear hearing device, Lyric2 (Phonak AG, Switzerland), which is the second generation of the Lyric device, featuring a smaller size in both length and diameter to increase the anatomic fitting rate. The study focused on three aspects: (a) integrity of the ear canal skin during the use of the extended-wear hearing device, (b) relationship between the integrity of the ear canal skin and continuation of wearing the device, and (c) potential change of the microbiological status in the skin flora with longer-term use.

\section{Materials and Methods}

\section{Subjects and Wearing Duration of Lyric2}

The study was approved by the local ethics committee (ref. No. KEK-ZH-Nr. 2011-0186). Fifty ears of 26 subjects ( 20 males and 6 females) with mild-to-moderate hearing loss were included in this study. For inclusion in the study, 40 subjects from the internal database of the validation team at Phonak AG were screened. They were chosen according to their hearing loss (they had to match the fitting range of Lyric2) and sized for Lyric2. If the sizing was successful, they were invited to participate in the study. The Lyric2 device was placed binaurally for 24 subjects and monaurally for 2 subjects who had a monaural hearing loss. The average age of the subjects was 59 years with a range from 22 to 87 years. The subjects were self-selected and received otolaryngological and audiological evaluation before the fitting, in order to confirm candidacy criteria [Arbogast, 2010]. These criteria included aspects of lifestyle (for instance, exclusion of scuba divers and sky divers), range of hearing loss, medical history (i.e. healthy outer and middle ear), and ear canal geometry. The Lyric2 is intended to be placed $4 \mathrm{~mm}$ from the tympanic membrane and initial placement complied with this requirement. However, slight changes of the device location were allowed at the time of the fitting if the change was helpful for the subject's comfort. Fittings were performed by audiologists in the audiology clinic at Phonak AG, who were trained for the fitting of the Lyric device. An ENT physician from the University Hospital Zurich also participated in the preinspection and in the examination of other aspects of the fitting.

Whenever the result of the first fitting was not satisfactory, a refitting was done if the subject agreed. The reasons for the refitting included: (a) technical issues of the device and (b) clinical issues such as the subject's discomfort and observation of signs of significant pathological conditions in the ear canal skin (grade 2; see the section 'Inspection of ear canal skin') as well as occlusion, migration of the device, and sound quality. While immediate and simple replacement of the device was chosen due to technical problems of the device, the refitting procedure for cases with clinical issues included inspection of ear canal integrity, change of the device size and/or readjustment of insert depth. When a replacement was due to a significant pathological condition in the ear canal skin with signs of grade 2 (table 1), device use was discontinued for 2 weeks and the pathological condition had to be resolved. Because the focus of this study was on the integrity and microbiological conditions of the ear canal and their clinical importance, simple replacement of the device due to technical issues was not included in the descriptive statistics concerning refitting due to clinical reasons. 

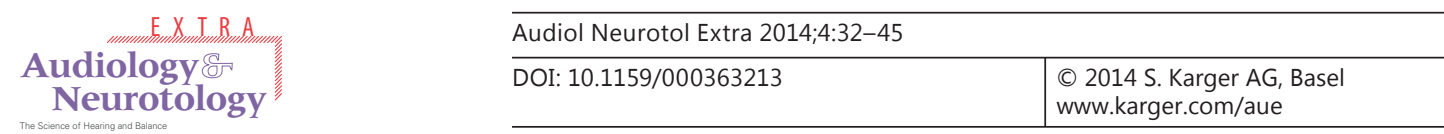

Sim et al.: Clinical and Microbiological Evaluation of an Extended-Wear Hearing Instrument
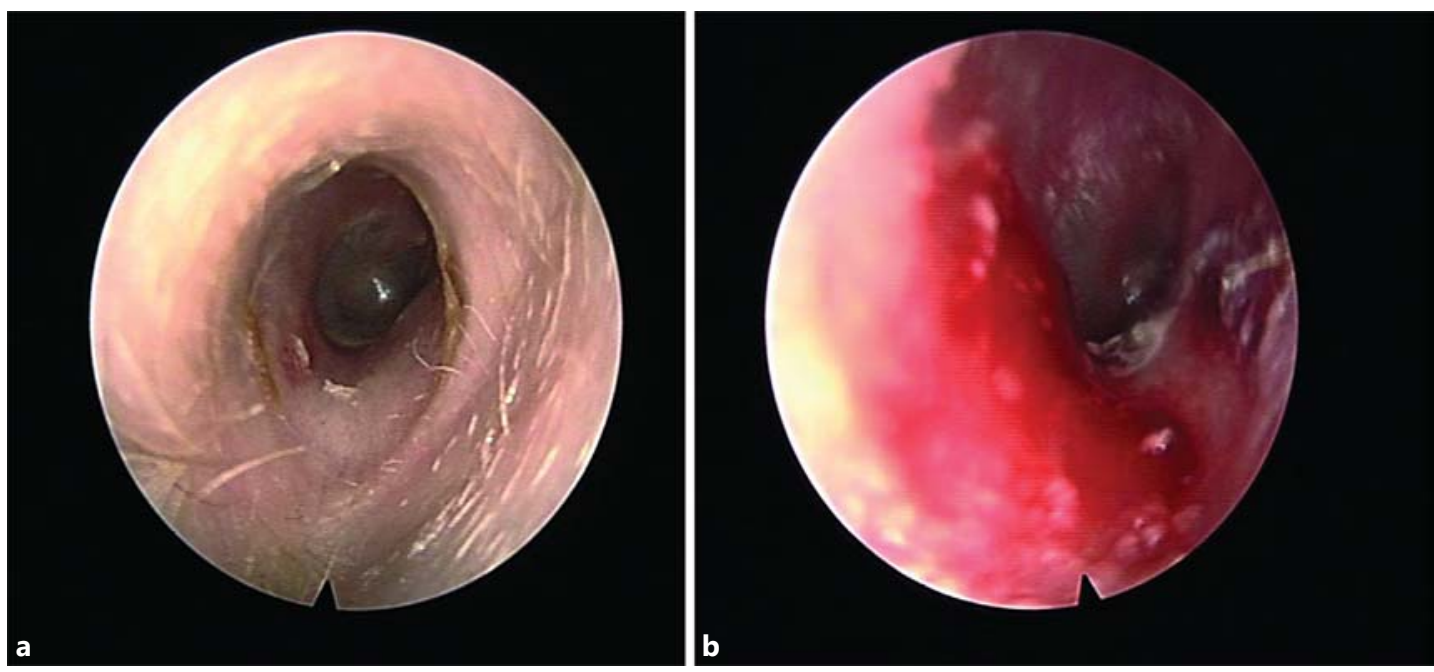

Fig. 2. Examination of pathological signs on the ear canal skin. a Ear canal skin with mild signs (grade 1; dry exudation and pressure mark observed). b Ear canal skin with severe signs (grade 2; moist exudation, significant edema, and ulcer observed).

Table 1. Pathological signs on the ear canal skin and their grade

\begin{tabular}{llll}
\hline Symptom & Grade & & \\
\cline { 2 - 3 } & 0 & 1 & 2 \\
\hline Erythema & none & mild & significant \\
Edema & none & mild & significant \\
Exudation & none & dry & moist \\
Ulcer & none & pressure mark & ulcer \\
\hline
\end{tabular}


with the first fitting but reached the required wearing duration with the refitting ('successful' with refitting). The 'unsuccessful' cases included those that failed with the first fitting and for whom refitting was not performed ('unsuccessful' without refitting) and cases who failed even with the refitting ('unsuccessful' with refitting). For all classifications (i.e. 'successful' without refitting, 'successful' with refitting, 'unsuccessful' without refitting, and 'unsuccessful' with refitting), the integrity of the ear canal skin (see section 'Inspection of ear canal skin') was analyzed as a factor determining continuation of device use.

\section{Microbiological Investigations}

Microbiological examinations were performed at three different stages: (a) prior to the fitting (on the same day as the fitting), (b) 10-14 days after the fitting (together with the 2nd inspection of ear canal skin pathology; see section 'Inspection of ear canal skin'), and (c) the end of the first period of wearing the device before it was changed - only for 'successful' cases (i.e. at least 60 days after the fitting; see section 'Correlation between ear canal skin integrity and continuation of wearing Lyric2'). Specimens were obtained from two locations in the ear canal with a commercial thin sterile transport swab that was inserted into the corresponding standard transport tube with Amies agar without charcoal (110C.USE AMIES W/O CH, Copan, Brescia, Italy). The first location was slightly toward a site lateral to the end of the Lyric2 device (denoted as 'lateral' location) and the second slightly medial to the medial end of the Lyric2 device (denoted as 'medial' location), thus corresponding to the outer and inner sides of the Lyric2 device as it sits in the ear canal. The device was removed and the auditory canals were swabbed gently by rolling the swabs $360^{\circ}$. The collected swabs were sent to the microbiological laboratory (Institute of Medial Microbiology, University of Zurich) within $8 \mathrm{~h}$. Before each swab, an otoscopic examination was performed to ensure that there was no active ear disease. The device was replaced after the specimens had been obtained.

Specimens were inoculated semiquantitatively onto Columbia sheep blood (5\%) agar plates (BioMérieux, Marcy l'Etoile, France), Columbia sheep blood (5\%) agar plates with colistin and nalidixic acid (BioMérieux) and MacConkey agar plates (BioMérieux). Growth of colonies was interpreted as little, moderate and numerous, but for the results, any growth of a species or a group of bacteria, i.e. coagulase-negative staphylococci or Corynebacterium spp., was equally registered. Bacteria were identified according to standard laboratory procedures by Versalovic et al. [2011].

The first microbiological test (prior to the fitting) was performed for 41 ears of 21 subjects because the required tests were not available when the study was started. Eight additional ears were evaluated at the second microbiological examination (10-14 days after the fitting). Among the 41 ears for which the first microbiological test was performed, 25 were still wearing the device at the second microbiological test, and 13 at the third microbiological test (more than 60 days after the fitting), without discontinuation of wearing the device. Among the 8 ears that were added to the second microbiological test, only 2 ears kept wearing the device without discontinuation and were evaluated at the third microbiological examination. As a consequence, all the 1st, $2 \mathrm{nd}$, and 3rd inspections were performed only for 13 ears, and 1 or 2 out of the 3 examinations were missed for other ears. The total range of the microbiological tests is illustrated in figure 3.

The same microbiological tests were also performed for the control group, which consisted of 10 subjects who were chosen among the R\&D crew at Phonak AG, and had no history of using any form of hearing aids for 3 months prior to obtaining the specimens. The average age of the control group members was 47 years (range from 35 to 59 years), and the gender distribution was 4 males and 6 females. The swab samples were taken from one side with the two different locations in the ear canal (lateral and medial), which corresponded to the same locations in the study group. The microbiological tests were performed only once for the control group. 

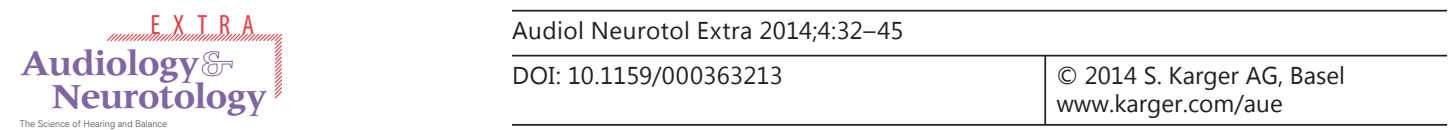

Sim et al.: Clinical and Microbiological Evaluation of an Extended-Wear Hearing Instrument

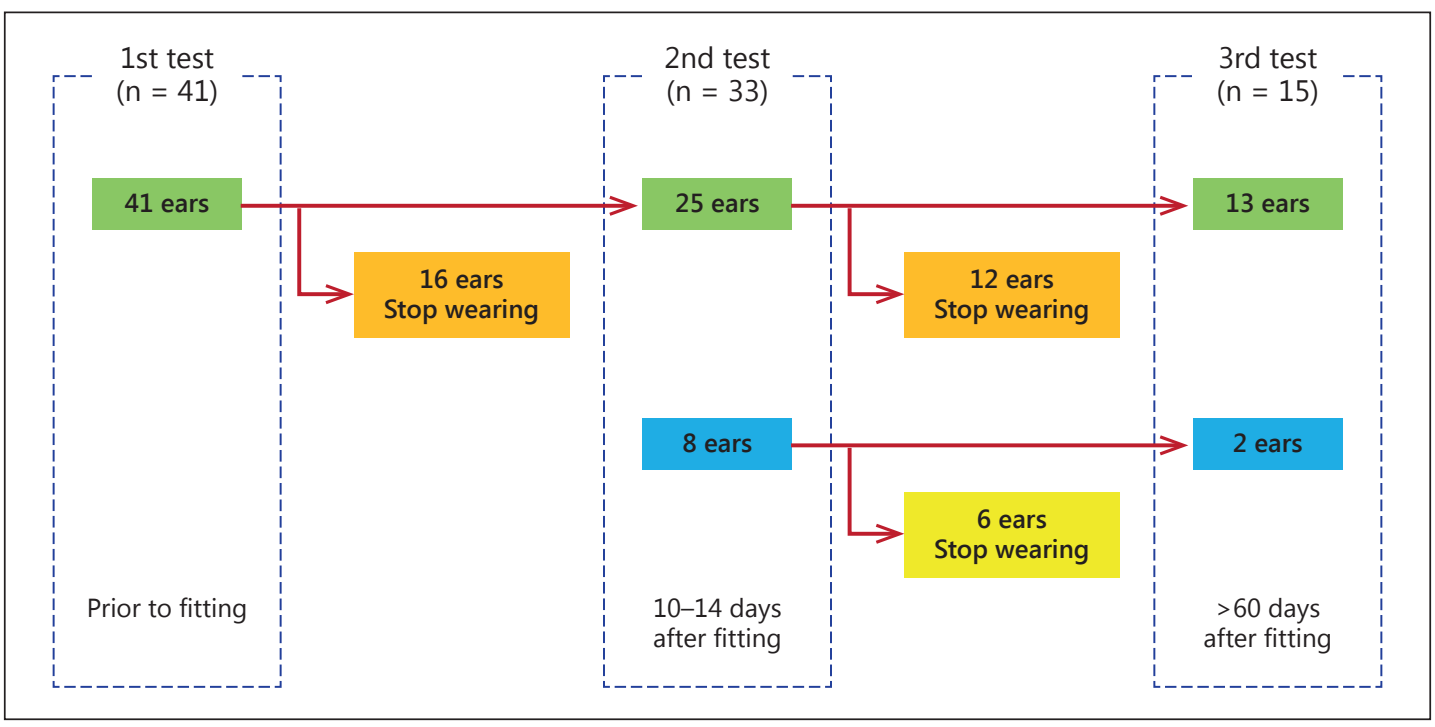

Fig. 3. Course of microbiological tests. The first microbiological test (prior to the fitting) was performed for 41 ears from 21 subjects, and 8 ears were added in the second microbiological examination (10-14 days after the fitting). All 3 inspections were only performed for 13 ears, and 1 or 2 of the 3 examinations were missing for the other ears because the subjects did not keep wearing the device.

Statistical calculation was performed for comparison of the results of the microbiological examinations between the different inspection periods and for comparison between each of the test periods in the study group and the control group. For each bacterium observed, the McNemar test was used for comparison between the different test periods in the study group, and the Fisher exact test was used for comparison between each of the test periods in the study group and the control group. Because the existence of Gram-negative bacteria was a focus in the microbiological investigation of the study (see Discussion), the specimens were classified into three categories: (a) existence of Gram-negative bacteria, (b) existence of Gram-positive bacteria only, and (c) no bacteria. The signed rank test was used for comparison between the different test periods for each of the classifications to determine development, and the Mann-Whitney test was used for comparison between the study group and control group. Differences with a p value of $<0.05$ were considered as the level of statistical significance.

\section{Results}

\section{Inspection of Ear Canal Skin}

Table 2 lists the results of the follow-up inspections of the ear canal during the first fitting and refitting of the devices, and long-term inspections in ears that were fitted successfully. During the first fitting, $75 \%$ of the inspected ears showed signs of erythema, edema, exudation, and/or ulcer in the 1st follow-up inspection (3-5 days after the first fitting), and such signs were observed in $55.2 \%$ of the inspected ears in the 2 nd follow-up inspection (10-14 days after the first fitting). The reduced percentage in the 2 nd follow-up inspection may be explained by the fact that those subjects who exhibited significant signs (grade 2 in table 1 ) or mentioned discomfort during the first inspection were told to stop using the device. Such a trend was also shown in the cases that were refit. During the refitting, these signs were 
Sim et al.: Clinical and Microbiological Evaluation of an Extended-Wear Hearing Instrument

Table 2. Results of ear canal skin inspections during the first fitting and refitting, and long-term inspections in subjects wearing the device successfully

\begin{tabular}{|c|c|c|c|c|c|}
\hline & \multicolumn{2}{|l|}{ 1st fitting } & \multicolumn{2}{|l|}{ Refitting } & \multirow{2}{*}{$\begin{array}{l}\text { Long- } \\
\text { term }{ }^{3}\end{array}$} \\
\hline & $\begin{array}{l}\text { 1st } \\
\text { inspection }^{1}\end{array}$ & $\begin{array}{l}\text { 2nd } \\
\text { inspection }{ }^{2}\end{array}$ & $\begin{array}{l}\text { 1st } \\
\text { inspection }^{1}\end{array}$ & $\begin{array}{l}\text { 2nd } \\
\text { inspection }^{2}\end{array}$ & \\
\hline Only one sign & $23(52.3)$ & $12(41.4)$ & $7(53.8)$ & $1(12.5)$ & $8(32.0)$ \\
\hline Erythema & $6(13.6)$ & $4(13.8)$ & $3(23.1)$ & $0(0)$ & $3(12.0)$ \\
\hline Edema & $0(0)$ & $0(0)$ & $3(23.1)$ & $0(0)$ & $0(0)$ \\
\hline Exudation & $12(27.3)$ & $6(20.7)$ & $0(0)$ & $1(12.5)$ & $5(20.3)$ \\
\hline Ulcer & $5(11.4)$ & $2(6.9)$ & $1(7.7)$ & $0(0)$ & $0(0)$ \\
\hline Combined signs & $10(22.7)$ & $4(13.8)$ & $2(15.4)$ & $2(25.0)$ & $0(0)$ \\
\hline Erythema + exudation & $4(9.1)$ & $2(6.9)$ & $0(0)$ & $1(12.5)$ & $0(0)$ \\
\hline Erythema + ulcer & $2(4.5)$ & $1(3.4)$ & $0(0)$ & $1(12.5)$ & $0(0)$ \\
\hline Exudation + ulcer & $1(2.3)$ & $1(3.4)$ & $1(7.7)$ & $0(0)$ & $0(0)$ \\
\hline Erythema + exudation + ulcer & $1(2.3)$ & $0(0)$ & $0(0)$ & $0(0)$ & $0(0)$ \\
\hline Edema + exudation + ulcer & $1(2.3)$ & $0(0)$ & $1(7.7)$ & $0(0)$ & $0(0)$ \\
\hline $\begin{array}{l}\text { Erythema + edema + } \\
\text { exudation + ulcer }\end{array}$ & $1(2.3)$ & $0(0)$ & $0(0)$ & $0(0)$ & $0(0)$ \\
\hline Nothing found & $11(25.0)$ & $13(44.8)$ & $4(30.8)$ & $5(62.5)$ & $17(68.0)$ \\
\hline Total inspected & $\begin{array}{l}44 \\
(6 \text { missed })\end{array}$ & $\begin{array}{l}29 \\
\text { (4 missed) }\end{array}$ & $\begin{array}{l}13 \\
(2 \text { missed })\end{array}$ & $\begin{array}{l}8 \\
(4 \text { missed })\end{array}$ & $\begin{array}{l}25 \\
(11 \text { missed })\end{array}$ \\
\hline
\end{tabular}

Figures in parentheses are percentages. Percentages were calculated with respect to the total number inspected.

${ }^{1}$ The 1 st follow-up inspections were done 3-5 days after the first fitting or refitting.

2 The 2nd follow-up inspections were done 10-14 days after the first fitting or refitting.

3 The long-term inspections were done only for those patients who used the device successfully.

observed in $69.2 \%$ of the 1 st follow-up inspections and in $37.5 \%$ of the $2 \mathrm{nd}$ follow-up inspections. At the end of the long-term use for ears successfully retaining the device, observation of pathological signs in the ear canal skin still occupied a larger portion (32\%), but all signs were mild (grade 1 in table 1).

Table 3 lists the classification of findings by grade $(0,1$, and 2$)$, where an ear was placed in the highest category if any physiological signs were observed, based on the severity of the condition. Such a classification was based on the fact that existence of grade 2 signs rather than the number of types of signs determined continuation of wearing the device. Grade 2 was observed most often in the 1st follow-up inspection after the initial fitting and was observed only in 1 ear in the first inspection during the refitting. Grade 2 was not observed in any of the long-term inspections. Grade 1 occupies the highest proportion except for the 2nd inspection during the refitting, where grade 0 (no symptom) was mostly observed. Overall, $95 \%$ of the ears during the various inspections were classified as grades 0 or 1 and were thus judged fit for immediate refitting and continuous use of the device, whereas in 5\%, there were observations with signs of grade 2 and the use of the device was interrupted.

The locations of the pathological signs in the ear canal as a function of the type of sign are shown in table 4. Erythema was seen mostly in the medial posterior region (17 ears), and also occurred considerably in the medial inferior region (10 ears). Edema was observed only in the medial posterior, medial inferior, and lateral posterior regions, but the trend could not be confirmed due to the small number of the samples (only in 3 ears). Exudation was distributed more evenly through the whole region of the ear canal while the region with the most effect was on the medial posterior surface. Signs of ulcers occurred mostly in the medial posterior 


\section{Audiology \\ Neurotology}

Table 3. Grades of pathological findings from inspection of the ear canal skin

\begin{tabular}{|c|c|c|c|c|}
\hline \multicolumn{5}{|c|}{ Audiol Neurotol Extra 2014;4:32-45 } \\
\hline \multicolumn{2}{|l|}{ DOI: $10.1159 / 000363213$} & \multicolumn{3}{|c|}{$\begin{array}{l}\text { (c) } 2014 \text { S. Karger AG, Basel } \\
\text { www.karger.com/aue }\end{array}$} \\
\hline \multicolumn{5}{|c|}{$\begin{array}{l}\text { Sim et al.: Clinical and Microbiological Evaluation of an Extended-Wear Hearing } \\
\text { Instrument }\end{array}$} \\
\hline \multirow[t]{2}{*}{ Inspection } & \multicolumn{3}{|c|}{ Number of samples } & \multirow{2}{*}{$\begin{array}{l}\text { Total } \\
\text { examined }\end{array}$} \\
\hline & grade 0 & grade 1 & grade 2 & \\
\hline \multicolumn{5}{|l|}{ First fitting } \\
\hline 1st inspection & $11(25.0)$ & $28(63.6)$ & $5(11.4)$ & 44 \\
\hline 2nd inspection & $13(44.8)$ & $16(55.2)$ & $0(0)$ & 29 \\
\hline \multicolumn{5}{|l|}{ Refitting } \\
\hline 1st inspection & $4(30.8)$ & $8(61.5)$ & $1(7.7)$ & 13 \\
\hline 2nd inspection & $5(62.5)$ & $3(37.5)$ & $0(0)$ & 8 \\
\hline Long-term inspection & $17(68.0)$ & $8(32.0)$ & $0(0)$ & 25 \\
\hline
\end{tabular}

Figures in parentheses are percentages. The grade was always determined as the highest grade when there was any ambiguity.

Table 4. Spatial distribution of the pathological signs of the ear canal skin problems

\begin{tabular}{|c|c|c|c|c|c|c|c|c|c|}
\hline & \multicolumn{9}{|c|}{ Number of observed samples } \\
\hline & \multicolumn{8}{|c|}{ location } & \multirow[t]{2}{*}{ ears with signs } \\
\hline & MA & MP & MS & MI & LA & LP & LS & LI & \\
\hline Erythema & 2 & 17 & 4 & 10 & 4 & 4 & 4 & 5 & $28 / 119(23.5 \%)$ \\
\hline Edema & 0 & 2 & 0 & 2 & 0 & 1 & 0 & 0 & $3 / 119(2.5 \%)$ \\
\hline Exudation & 8 & 17 & 7 & 11 & 5 & 7 & 5 & 8 & $40 / 119(33.6 \%)$ \\
\hline Ulcer & 1 & 7 & 1 & 7 & 4 & 2 & 1 & 4 & $21 / 119(17.6 \%)$ \\
\hline
\end{tabular}

$\mathrm{MA}=$ Medial anterior; $\mathrm{MP}=$ medial posterior; $\mathrm{MS}=$ medial superior; $\mathrm{MI}=$ medial inferior; $\mathrm{LA}=$ lateral anterior; $\mathrm{LP}=$ lateral posterior; $\mathrm{LS}=$ lateral superior; $\mathrm{LI}=$ lateral inferior.

and medial inferior regions (7 ears for each region), and were also seen considerably in the lateral anterior and lateral inferior regions (4 ears for each region). The most common location overall was the medial posterior region, especially in the 1st inspection for both the first fitting and refitting.

\section{Ear Canal Skin Integrity as a Factor Determining Continuation of the Wearing}

Overall, 36 (69.2\%) ears were 'successful' cases without refitting (24 ears) and with refitting (12 ears), and 14 (30.8\%) were 'unsuccessful' without refitting (11 ears) and with refitting (3 ears) (fig. 4). The reasons for interruptions of the wearing after the initial fitting (26 ears) were: (1) issues of the ear canal skin (19 ears; 73.1\%), which also included 2 ears from 2 subjects who initially wore the device on both sides and had problems only on one side, but did not want to continue wearing the device unilaterally, (2) migration of the device ( 2 ears; $7.7 \%$ ), (3) sound quality and feedback (4 ears; 15.4\%), and (4) occlusion (1 ear; $3.8 \%)$. Therefore, problems with the ear canal skin were responsible for approximately two thirds of the first fitting failures. Among the 19 ears that had issues related to the ear canal skin, the associated subjects of 15 of these ears complained of discomfort and had corresponding issues related to the ear canal skin. However, the device had to be removed because of issues of the ear canal skin without reports of discomfort in 4 cases (3 ears, grade 2; 1 ear, grade 1 and the subject requested that the device be removed). There was no correlation between the subjects' feelings of discomfort and the type of pathological signs in the ear canal. 

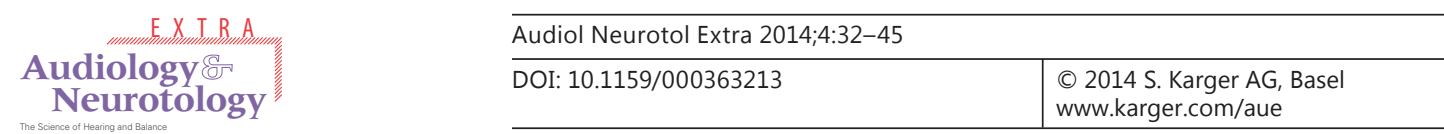

Sim et al.: Clinical and Microbiological Evaluation of an Extended-Wear Hearing Instrument

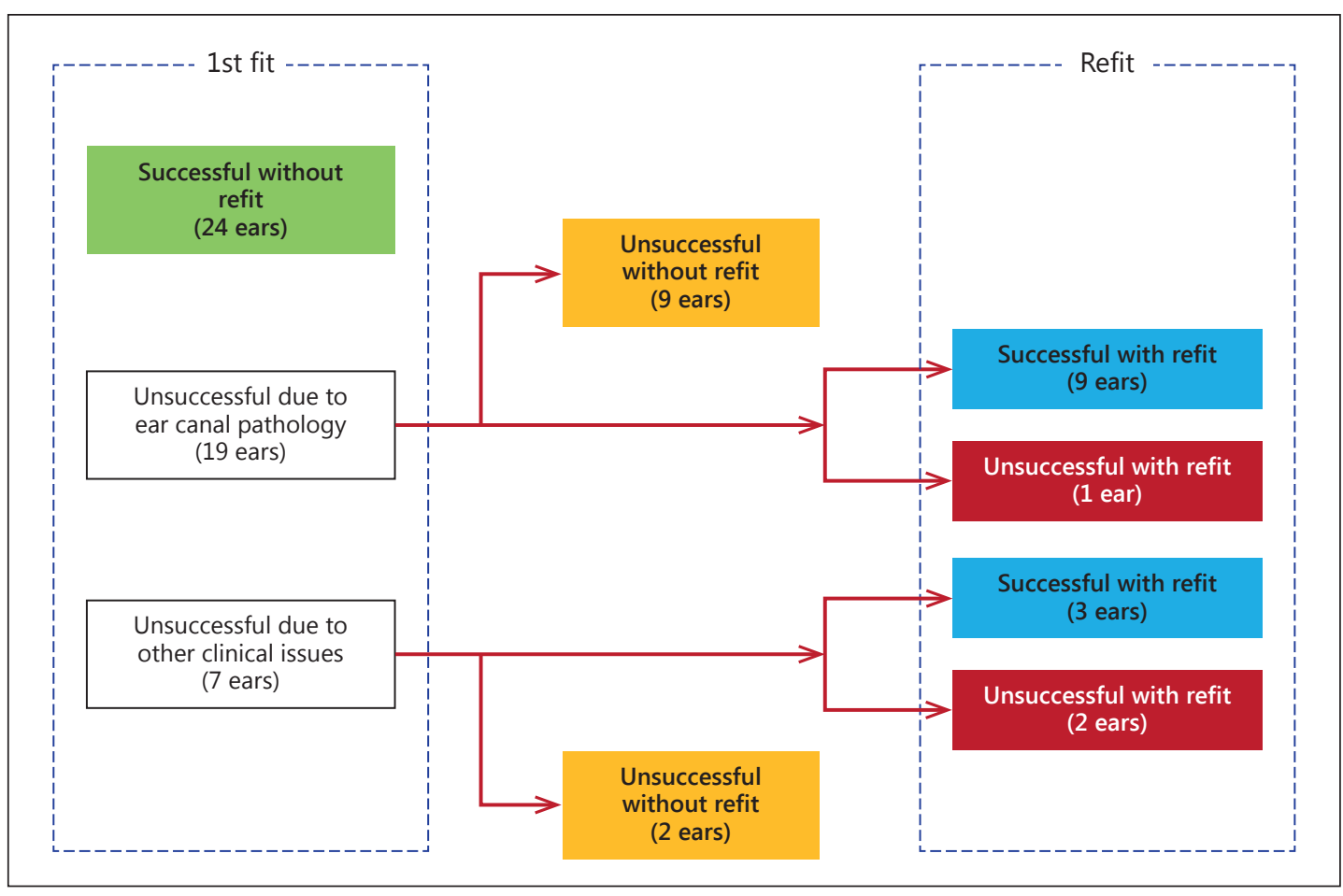

Fig. 4. 'Successful' and 'unsuccessful' cases of use of the Lyric2 device ( $n=50)$. The problematic conditions of the ear canal pathology were responsible for 9 of 11 'unsuccessful' cases without refitting and for 1 of 3 'unsuccessful' cases with refitting.

Refitting was done for 15 of 26 ears in which the first fitting was interrupted. There was no refitting for the other 11 ears $(22 \%$ of the 50 total investigated ears, 'unsuccessful' cases without refitting) because the subjects did not want to continue wearing the device. With the refitting, 12 ears ( $80 \%$ of the 15 refitted and $24 \%$ of the 50 total investigated ears, 'successful' cases with refitting) were 'successful', and wearing the device was interrupted again for 3 ears $(25 \%$ of the 15 refitted and $6 \%$ of the 50 total investigated ears, 'unsuccessful' cases with refitting). The reasons for the interruptions even with the refitting were pathological issues of the ear canal skin $(n=1)$, migration $(n=1)$, and occlusion $(n=1)$.

\section{Results of Microbiological Testing}

Tables 5 and 6 represent bacteria found in microbiological tests of specimens taken from the lateral (outer) and medial (inner) sides of the external ear canal. On both sides, coagulasenegative staphylococci were found most frequently not only in the study group, regardless of the duration of wearing the device, but also in the control group. No statistically significant difference was found between the different test periods in the study group or between the study group and the control group. Turicella otitidis also had a large number of occurrences in specimens from the 1st examination of the study group and the control group, but was found less often in the 2 nd and 3rd examinations of the study group. A statistically significant difference was found only between the 2 nd examinations of the study group and the control group, for the outer side ( $\mathrm{p}<0.05$, tested by Fisher's exact test). Other Gram-positive bacteria were observed, but their occurrence was much lower than those of coagulase-negative staphylococci and T. otitidis, without any statistically significant difference between the different test periods in the study group or between the study group and the control group. Some 
Sim et al.: Clinical and Microbiological Evaluation of an Extended-Wear Hearing Instrument

Table 5. Bacteria observed in microbiological tests at the lateral location (outer side of Lyric2)

\begin{tabular}{|c|c|c|c|c|}
\hline \multirow[t]{2}{*}{ Bacteria } & \multicolumn{3}{|c|}{ Study group } & \multirow{2}{*}{$\begin{array}{l}\text { Control } \\
\text { group } \\
(\mathrm{n}=10)\end{array}$} \\
\hline & $\begin{array}{l}\text { 1st test } \\
(n=41)\end{array}$ & $\begin{array}{l}\text { 2nd test } \\
(n=33)\end{array}$ & $\begin{array}{l}3 r d \text { test } \\
(n=15)\end{array}$ & \\
\hline \multicolumn{5}{|l|}{ Gram-positive } \\
\hline Coagulase-negative staphylococci & $30(73.2)$ & $19(57.6)$ & $10(66.7)$ & $8(80.0)$ \\
\hline Turicella otitidis & $11(26.8)$ & $3(9.1)$ & $1(6.7)$ & $4(40.0)$ \\
\hline Corynebacterium sp. & $2(4.9)$ & $0(0)$ & $2(13.3)$ & $1(10.0)$ \\
\hline Staphylococcus aureus & $2(1.9)$ & $0(0)$ & $0(0)$ & $1(10)$ \\
\hline Lactobacillus sp. & $1(2.4)$ & $0(0)$ & $0(0)$ & $0(0)$ \\
\hline Bacillus sp. & $0(0)$ & $0(0)$ & $1(6.7)$ & $0(0)$ \\
\hline Viridans streptococci & $0(0)$ & $0(0)$ & $0(0)$ & $1(10.0)$ \\
\hline Micrococcus luteus & $0(0)$ & $0(0)$ & $0(0)$ & $1(10.0)$ \\
\hline \multicolumn{5}{|l|}{ Gram-negative } \\
\hline Acinetobacter sp. & $1(2.4)$ & $0(0)$ & $0(0)$ & $0(0)$ \\
\hline Klebsiella oxytoca & $1(2.4)$ & $0(0)$ & $0(0)$ & $0(0)$ \\
\hline Brevundimonas diminuta/vesicularis & $1(2.4)$ & $0(0)$ & $0(0)$ & $0(0)$ \\
\hline Flavimonas oryzihabitans & $0(0)$ & $0(0)$ & $0(0)$ & $0(0)$ \\
\hline Pseudomonas aeruginosa & $0(0)$ & $0(0)$ & $0(0)$ & $0(0)$ \\
\hline
\end{tabular}

Figures in parentheses are percentages.

Table 6. Bacteria observed in microbiological tests at the medial location (inner side of Lyric2)

\begin{tabular}{|c|c|c|c|c|}
\hline \multirow[t]{2}{*}{ Bacteria } & \multicolumn{3}{|c|}{ Study group } & \multirow{2}{*}{$\begin{array}{l}\text { Control } \\
\text { group } \\
(\mathrm{n}=10)\end{array}$} \\
\hline & $\begin{array}{l}\text { 1st test } \\
(n=41)\end{array}$ & $\begin{array}{l}2 \text { nd test } \\
(n=33)\end{array}$ & $\begin{array}{l}\text { 3rd test } \\
(n=15)\end{array}$ & \\
\hline \multicolumn{5}{|l|}{ Gram-positive } \\
\hline Coagulase-negative staphylococci & $27(65.9)$ & $16(48.5)$ & $9(60.0)$ & $7(70.0)$ \\
\hline Turicella otitidis & $10(24.4)$ & $2(6.1)$ & $1(6.7)$ & $3(30.0)$ \\
\hline Corynebacterium sp. & $1(2.4)$ & $1(3.0)$ & $1(6.7)$ & $1(10.0)$ \\
\hline Staphylococcus aureus & $1(2.4)$ & $0(0)$ & $0(0)$ & $1(10)$ \\
\hline Lactobacillus sp. & $1(2.4)$ & $0(0)$ & $0(0)$ & $0(0)$ \\
\hline Bacillus sp. & $0(0)$ & $0(0)$ & $0(0)$ & $0(0)$ \\
\hline Viridans streptococci & $0(0)$ & $0(0)$ & $0(0)$ & $0(0)$ \\
\hline Micrococcus luteus & $0(0)$ & $0(0)$ & $0(0)$ & $1(10.0)$ \\
\hline \multicolumn{5}{|l|}{ Gram-negative } \\
\hline Acinetobacter sp. & $0(0)$ & $0(0)$ & $0(0)$ & $0(0)$ \\
\hline Klebsiella oxytoca & $0(0)$ & $0(0)$ & $0(0)$ & $0(0)$ \\
\hline Brevundimonas diminuta/vesicularis & $0(0)$ & $0(0)$ & $0(0)$ & $0(0)$ \\
\hline Flavimonas oryzihabitans & $1(2.4)$ & $0(0)$ & $0(0)$ & $0(0)$ \\
\hline Pseudomonas aeruginosa & $0(0)$ & $0(0)$ & $0(0)$ & $1(10.0)$ \\
\hline
\end{tabular}

Figures in parentheses are percentages.

Gram-negative bacteria were found in a few ears of subjects in the control group and in the 1st examination of the study group but not in the 2 nd and $3 \mathrm{rd}$ examinations of the study group. No statistically significant difference was detected between the different test periods in the study group or between the study group and the control group, for any of the Gramnegative bacteria. 


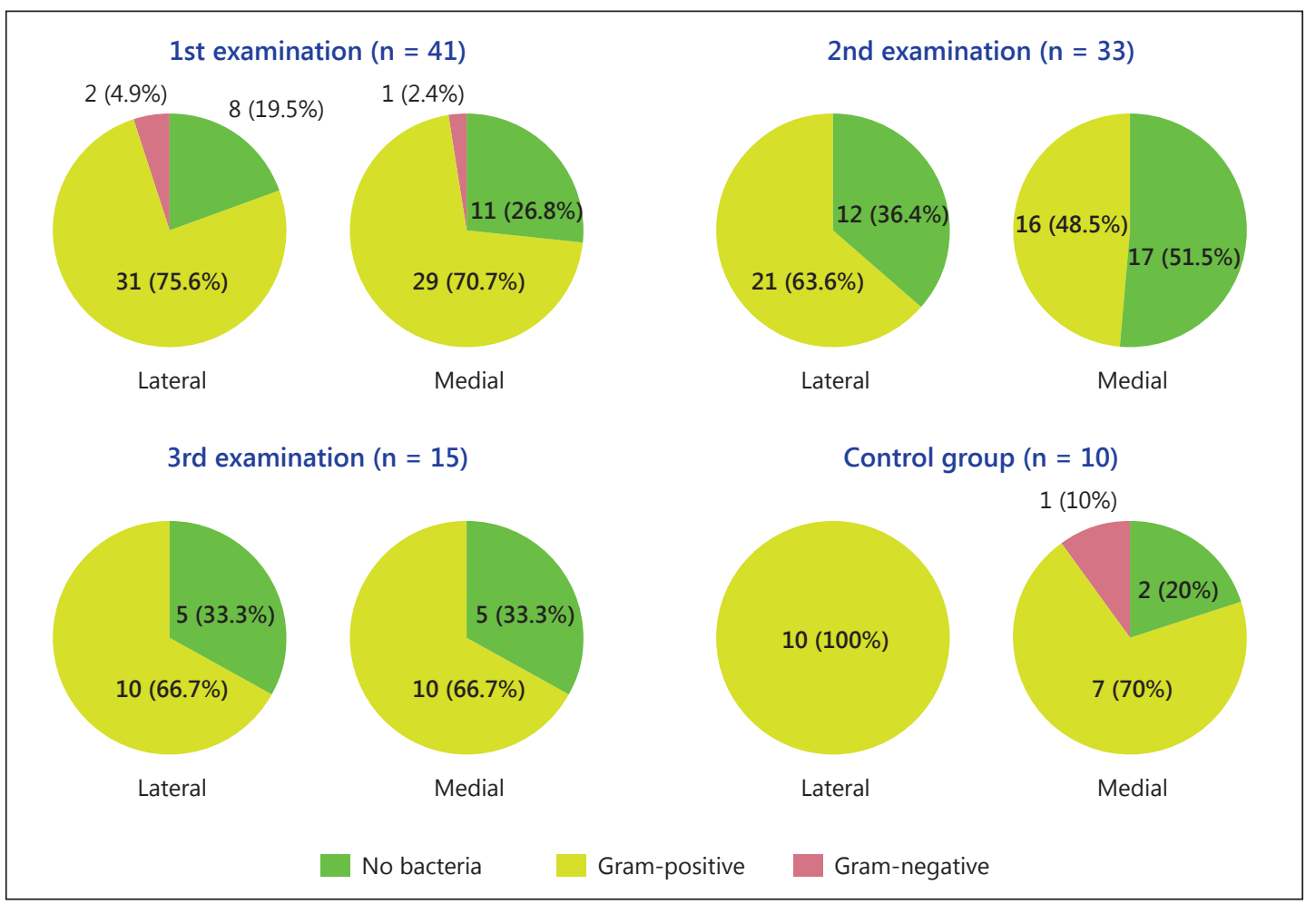

Fig. 5. Microbiological status at each of the test periods in the study group and in the control group.

Figure 5 displays the distribution of the microbiological statuses at the different test periods in the study group and in the control group, with classification of the microbiological statuses into 'no bacteria', 'Gram-positive' (i.e. Gram-positive only), and 'Gram-negative' according to types of bacteria observed. The status of 'Gram-positive' occupied the largest portion in both the study group (overall, $69.7 \%$ for the lateral location and $61.8 \%$ for the medial location) and the control group (100\% for the lateral side and $70 \%$ for the medial side). The status of 'Gram-negative' was found only at the 1st examination and in only 3 samples from 2 ears (observed in both the lateral and medial sides for one ear and only in the lateral side for the other ear), in the study group. No Gram-negative bacteria were found in the 2nd and 3rd examinations in the study group. In the control group, a Gram-negative bacterium of Pseudomonas aeruginosa was observed only in 1 sample (medial side). Comparison between different test periods in the study group revealed no development from 'no bacteria' to 'Grampositive' or 'Gram-negative' and from 'Gram-positive' to 'Gram-negative'. In the comparison between each of the test periods in the study group and the control group, the 2 nd test period of the study group had a significantly larger portion of 'no bacteria' than that of the control group, for both outer and inner sides ( $\mathrm{p}<0.05$, Mann-Whitney test).

\section{Discussion}

The clinical safety of the first generation of the Lyric device was first assessed by Scherl et al. [2011] in a retrospective study. In that study, longitudinal data from 364 subjects wearing the device over the course of up to 2 years were gathered and examined, concluding that the device was safe if the candidacy guidelines and sizing procedures [Arbogast and Whichard, 
Sim et al.: Clinical and Microbiological Evaluation of an Extended-Wear Hearing Instrument

2009; Arbogast, 2010] were followed well and the fitters properly trained. In our study with the second generation of the device, clinical safety was examined in greater detail, focusing primarily on the integrity and microbiological aspects of the ear canal skin. A potential bias in our approach of including patients could be the difference in motivation. The subjects who are willing to participate in studies to test new technical developments are not necessarily motivated by the same reasons as individuals in need for a solution to their hearing loss.

Overall, $95 \%$ of the inspected ears in the study group showed no (grade 0 ) or mild (grade 1) signs with $5 \%$ showing grade 2 signs (table 3 ). These results indicate that the Lyric2 can be judged suitable for immediate refitting and continuous use in most cases with respect to ear canal skin integrity. The loci of the pathological signs (table 4) indicated that the medial posterior region of the ear canal is the most vulnerable area for effects related to its insertion or wear. Further improvement in the insertion process and/or design of the device may be needed to avoid problematic conditions of the ear canal skin physiology in this region. It has been observed that ear canal skin integrity is usually disrupted within the first 30 days of wearing the Lyric2. If patients show no such issues after 30 days, they are not likely to develop skin alteration later on. As most grade 2 findings are observed around the medial end of the device in the bony part of the ear canal, the patients were offered to wear a standard hearing aid if the ENT specialist gave clearance and they possessed a hearing aid. Given the motivation of most Lyric2 customers for the product (invisibility and no handling), a standard hearing aid was not appreciated by most of the subjects.

Though wearing the Lyric2 does not cause problems of the ear canal skin in most cases, this is still considered as the main reason why users must stop wearing the device. Two thirds of the unsuccessful cases in the first fitting category (19 out of 26) were caused by issues related to the skin of the ear canal. Additionally, some subjects who experienced problematic conditions with ear canal skin did not want to continue with a refitting and chose to quit wearing the device when the first stage was completed ( 9 ears from 6 subjects). In the category of the 'unsuccessful', which included those with refitting $(n=3)$ and without refitting $(n=11)$, pathological conditions of the ear canal skin were responsible for $71.4 \%$ of the unsuccessful ears $(1 / 3$ 'unsuccessful' with refitting, 9/11 'unsuccessful' without refitting; thus, 10 of 14 ears were categorized as 'unsuccessful' due to issues of the ear canal skin).

However, from the results following refitting, interruption from wearing the device reduced the pathological conditions of the ear canal skin after the device was fitted more carefully. Among 10 ears (of 6 subjects) that were unsuccessful with the first fitting and were subsequently refitted, 9 were successful and only 1 remained unsuccessful. When the 11 unsuccessful fittings that were not refit are excluded, then only $1 / 39$, approximately $2.5 \%$ of the investigated ears, was unsuccessful due to ear canal skin problems. Such a result reveals that wearing the device can be more successful in the initial fitting if the size of the device is chosen more appropriately and the fitting is done more carefully. The outcome of fitting the Lyric2 device can be considered to be highly dependent upon the experience and careful training of the individual placing the device.

The incidence of bacteria in the external auditory canal is common even in healthy conditions, and bacteria found in the healthy ears are mostly Gram-positive. In a study by Stroman et al. [2001], swab specimens were collected from 164 healthy subjects, and growth of bacteria was absent in only 17 subjects. A total of 302 bacteria from external ear swabs of 147 ears were found, and $96 \%$ of the 302 bacteria were Gram-positive. Staphylococci were present in $63 \%$. The coryneform bacteria represented $19.5 \%$ of the bacteria found, with T. otitidis detected in $12 \%$ and Corynebacterium auris in $2.5 \%$. The third most frequent bacteria belonged to the streptococci and enterococci group with $7 \%$ of all bacteria. Campos et al. [1998] also showed similar results. While several types of Gram-positive bacteria are common even in healthy ears, these bacteria are regarded not to be harmful to immune-competent 
subjects. The existence and abundance of such Gram-positive bacteria in the skin flora of the ear canal are determined by the subjects' living and working environment. The bacteria found in the external auditory canal before the placement of the Lyric2 device (1st test in tables 5 and 6) represent the normal bacterial flora, which can also be observed in microorganisms of ears in the control group. Infections of the external auditory canal are polymicrobial in $50 \%$ of cases and mostly caused by aerobic bacteria, which are Gram-negative. The most often isolated bacteria in infected external auditory canals are P. aeruginosa and Staphylococcus spp. [Brook et al., 1992; Agger and Mardan, 1995; Amigot et al., 2003; Schaefer and Baugh, 2012; Lee et al., 2013]. These bacteria could not be cultured in any swabs after placement of the Lyric2 device in our study.

Predisposing factors for infections of the external auditory canal are disruption of the integrity of the skin due to trauma, dermatologic diseases (i.e. eczema, psoriasis), increase of pH [Lee et al., 2013], and increased humidity of the canal causing maceration of the epithelial lining [Kowalski, 1988]. An increase in humidity would have favored the growth of Gramnegative rods, especially P. aeruginosa [Seyfried and Fraser, 1978; Brook and Coolbaugh, 1984]. A recent study by Karaca et al. [2013] found significantly more positive bacterial cultures in the ear with a hearing aid (61\%) as compared to the contralateral ear without a hearing aid (3\%) in the same subject. The study concluded that occlusion of the canal by hearing aids may make the ear canal become a warmer and moist environment and may cause change of the $\mathrm{pH}$ of cerumen as well, providing favorable conditions for bacteria growth. However, in our study, an increase of bacteria after placement of the Lyric2 device or a significant difference of the bacteria between the study group (i.e. ears with Lyric2) and the control group (i.e. ears without Lyric2) was not observed. A possible explanation for the discrepancy between the two studies is that occlusion by different types of hearing aids may result in different physiological conditions of the ear canal such as temperature, humidity, and pH. For instance, the amount of humidity transport through a hearing aid can be different depending upon the material of the ear mold or the hearing aid, the size of venting, and the amount of occluded space. As the humidity and $\mathrm{pH}$ of the ear canal were not measured in either study, this possibility cannot be assessed. However, considering the results of our study, it is presumed that a significant change of the ear canal environment is not caused by wearing the Lyric2. Another possibility is that outpouring of resident bacteria from the deeper skin layers, sweat, and sebaceous glands is more significant for the use of conventional hearing aids as in the study by Karaca et al. [2013] than for the use of Lyric2 in this study. While the Lyric2 device is placed deep in the ear canal by trained audiologists and remains in situ for a maximum of 120 days until the battery is depleted and the device is replaced, conventional hearing aids are inserted and removed frequently on a day-to-day basis. Therefore, risks of contact transmission of microorganisms are smaller for the Lyric2 device. Contact transmission occurs when a subject removes/inserts a hearing aid after contacting a contaminated area without properly washing her/his hands. Somewhat surprising is that trauma of the ear canal skin, a known risk factor for external otitis [Hawke et al., 1984], induced by the Lyric2 device in some subjects seems not to induce bacterial growth. Subjects discontinued wearing the Lyric2 device when there was painful skin irritation.

\section{Conclusion}

The main objective of this study was to examine the integrity and microbiological changes of the ear canal skin caused by wearing Lyric2, an extended-wear hearing device.

From the inspection of the integrity of the ear canal skin, the Lyric 2 can be judged suitable for immediate refitting and continuous use in most cases. Ninety-five percent were judged to 
Sim et al.: Clinical and Microbiological Evaluation of an Extended-Wear Hearing Instrument

be suitable for immediate refitting and continuous use of the device, whereas in $5 \%$, issues related to the ear canal skin pathology caused an interruption of use for the ear canal to recover.

Though wearing Lyric2 does not cause problems with ear canal skin pathology in most cases, this is still considered to be the main reason for interruption of wearing the device. Nevertheless, only $1 / 10$ ears that were unsuccessful in the first fitting due to issues related to pathology of the ear canal skin was unsuccessful again in the refitting, and the other 9 ears were successful. Considering the fact that refitting was done only with a change of the device's size and/or proper relocation, it is expected that the first fitting can be more successful if it is done more carefully using a properly sized device and by developing improved methods to minimize trauma during insertion.

The microbiological tests at three different stages of the use period revealed that occlusion by the device does not enhance or prompt the growth of Gram-negative bacteria, which can cause ear health-related issues. Therefore, extended wearing of the Lyric2 device can be considered safe from a microbiological perspective.

\section{Acknowledgements}

We thank the Division of Biostatistics of the University of Zurich for assisting in the statistical calculations in this study. This work was financially supported by Phonak AG, and was performed as a joint project of the University Hospital Zurich and Phonak AG.

\section{References}

-Agger WA, Mardan A: Pseudomonas aeruginosa infections of intact skin. Clin Infect Dis 1995;20:302-308.

Amigot SL, Gomez CR, Luque AG, Ebner G: Microbiological study of external otitis in Rosario City, Argentina. Mycoses 2003;46:312-315.

Arbogast T: Candidacy and fitting protocols for a 24/7 hearing device. Hear Rev 2010;17:34-37.

Arbogast T, Whichard S: A new hearing aid class: the first 100\% invisible extended-wear hearing aid. Hear Rev 2009;16:20-27.

Branda E: Deep canal fittings: advantages, challenges, and a new approach. Hear Rev 2012;19:24-27.

Brook I, Coolbaugh JC: Changes in the bacterial flora of the external ear canal from the wearing of occlusive equipment. Laryngoscope 1984;97:963-965.

Brook I, Frazier EH, Thompson DH: Aerobic and anaerobic microbiology of external otitis. Clin Infect Dis 1992;15: 955-958.

Campos A, Arias A, Betancor L, Rodríguez C, Hernández AM, López Aguado D, Sierra A: Study of common aerobic flora of human cerumen. J Laryngol Otol 1998;112:613-616.

Gudmundsen G: Fitting CIC hearing aids - some practical pointers. Hear J 1994;47:10, 45-48.

Hawke M, Wong J, Krajden S: Clinical and microbiological features of otitis externa. J Otolaryngol 1984;13:289-295.

Karaca ÇT, Akçay SŞ, Toros SZ, Oysu Ç, Verim A, Çelebi Ş, Aksaray S: External auditory canal microbiology and hearing aid use. Am J Otolaryngol 2013;34:278-281.

Kochkin S: MarkeTrak VII: obstacles to adult non-user adoption of hearing aids. Hear J 2007;60:24-50.

Kowalski JJ: The microbial environment of the ear canal in health and disease. Vet Clin North Am Small Anim Pract 1988;18:743-754.

Lee H, Kim J, Nguyen V: Ear infections: otitis externa and otitis media. Prim Care 2013;40:671-686.

Schaefer P, Baugh RF: Acute otitis externa: an update. Am Fam Physician 2012;86:1055-1061.

Scherl M, Szabo D, Desai N, Scherl S, Arbogast T: Real-world safety experience with a 24/7 hearing device. Hear Rev 2011;18:18-23.

- Seyfried PL, Fraser DJ: Pseudomonas aeruginosa in swimming pools related to the incidence of otitis externa infection. Health Lab Sci 1978;15:50-57.

Stroman DW, Roland PS, Dohar J, Burt W: Microbiology of normal external auditory canal. Laryngoscope 2001;111: 2054-2059.

Versalovic J, Carroll KC, Funke G, Jorgensen JH, Landry ML, Warnock DW: Manual of Clinical Microbiology, ed 10. Washington, ASM Press, 2011. 